\title{
Attenuation in Superconducting Circular Waveguides
}

\author{
Kim Ho Yeap ${ }^{1}$, Sing Sean Ong ${ }^{1}$, Humaira Nisar ${ }^{1}$, \\ Koon Chun Lai ${ }^{1}$, and Choon Aun $\mathrm{Ng}^{1}$ \\ ${ }^{1}$ Faculty of Engineering and Green Technology, Tunku Abdul Rahman University, Jalan Universiti, Bandar Barat, 31900 \\ Kampar, Perak. Malaysia. \\ *corresponding author, E-mail: yeapkh@utar.edu.my
}

\begin{abstract}
We present an analysis on wave propagation in superconducting circular waveguides. In order to account for the presence of quasiparticles in the intragap states of a superconductor, we employ the characteristic equation derived from the extended Mattis-Bardeen theory to compute the values of the complex conductivity. To calculate the attenuation in a circular waveguide, the tangential fields at the boundary of the wall are first matched with the electrical properties (which includes the complex conductivity) of the wall material. The matching of fields with the electrical properties results in a set of transcendental equations which is able to accurately describe the propagation constant of the fields. Our results show that although the attenuation in the superconducting waveguide above cutoff (but below the gap frequency) is finite, it is considerably lower than that in a normal waveguide. Above the gap frequency, however, the attenuation in the superconducting waveguide increases sharply. The attenuation eventually surpasses that in a normal waveguide. As frequency increases above the gap frequency, Cooper pairs break into quasiparticles. Hence, we attribute the sharp rise in attenuation to the increase in random collision of the quasiparticles with the lattice structure.
\end{abstract}

\section{Introduction}

Circular waveguides have been widely used in radio telescopes to channel signals to the receiver circuits. The front end receiver noise temperature is determined by a number of factors. These include the mixer noise temperature $T_{M}$, the conversion loss $C_{\text {loss }}$, the noise temperature of the first $I F$ amplifier $T_{F}$ and the coupling efficiency between the $I F$ port of the junction and the input port of the first $I F$ amplifier $\eta_{I F}$. Walker et al. [1] have performed a comparison among different waveguide receivers. It was found that the deterioration in system performance is partly affected by the increase in conversion loss $C_{\text {loss. }}$. Since signals from distant sources are usually extremely faint, it is therefore important to ensure that the conversion loss $C_{\text {loss }}$ of the mixer circuit could be kept to its minimal. To minimize the loss of the signals, the availability of a highly efficient waveguide is certainly central to the development of the receiver circuit [2].

Most waveguides implemented in the receiver system are fabricated using copper. Due to the weak intensity of the signals, however, the attenuation level in standard metallic waveguides may actually cause significant degradation to the signals [3]. Superconductors are known to feature low loss. It is, therefore, interesting to perform an investigation on wave propagation in superconducting circular waveguides.

In [4] and [5], analysis on the performance of superconducting circular waveguides has been performed based on Mattis-Bardeen theory. Since the equations are derived from Bardeen, Cooper and Schrieffer BCS weak coupling theory, it takes into account the presence of the gap energy. According to the BCS theory, the electronic states in the immediate vicinity of the Fermi energy $E_{F}$ have their energy pushed away from $E_{F}$ [6]. Hence, no quasiparticle state exists within the gap energy. Recent findings show, however, that this may not be true. Experimental measurements actually suggested that intragap states exist within the gap energy [7] - [11].

In [10] and [11], Noguchi et al. have modified MattisBardeen theory to account for the presence of the intragap states. Measurements on the surface resistance of the superconductor were found to agree with those computed using this extended Mattis-Bardeen theory. Since the new equations are able to give a more realistic behavior of a superconductor, we have applied them in [12] to analyze wave propagation in superconducting rectangular waveguides. Here, we extend further our approach to the case of a superconducting circular waveguide. We apply the complex conductivity of a superconductor, derived using the extended Mattis-Bardeen theory, onto the equations presented in [5] which calculate the attenuation constant of a circular waveguide. In order to present a complete scheme, we briefly outline the extended Mattis-Bardeen theory and the characteristic equations in [5] in the following sections.

\section{Superconducting complex conductivity}

Due to the existence of the gap energy $2 \Delta(T)$ in a superconductor, the conductivity of the material is complex and can be expressed as 


$$
\sigma=\sigma_{1}-j \sigma_{2}
$$

where $\sigma_{1}$ and $\sigma_{2}$ represent the quasiparticle and Cooper-pair currents in a superconductor, respectively [10].

In order to take into account the existence of the intragap states, Noguchi et al. suggested to express the gap energy as a complex variable, i.e. $\Delta=\Delta_{1}+\mathrm{j} \Delta_{2}$, where $\Delta_{1}$ and $\Delta_{2}$ are real [10], [11]. Here, $\Delta_{1}$ can be expressed with the real gap energy given below $[12,13]$

$$
\begin{aligned}
\ln \left(\tilde{\Delta}_{1}\right) & =-2 \int_{0}^{\infty}\left(E^{2}+\tilde{\Delta}_{1}^{2}\right)^{-1 / 2} \times \\
& \left\{1+\exp \left[\left(\pi / \gamma_{E} \tilde{T}\right)\left(E^{2}+\tilde{\Delta}_{1}^{2}\right)^{1 / 2}\right]\right\}^{-1} d E
\end{aligned}
$$

where $\tilde{\Delta}_{1}=\Delta_{1}(T) / \Delta_{1}(0), \tilde{T}=T / T_{c}, T$ is the operating temperature, $T_{c}$ the critical temperature of the superconductor and $\gamma_{E}=1.781$ is the Euler's constant. By extending Mattis-Bardeen theory, the new complex conductivity $\sigma$ is derived as follows [12]

$$
\begin{aligned}
& \frac{\sigma}{\sigma_{n}}=\int_{\Delta_{1}}^{\infty} \frac{2\left[f\left(E_{r}\right)-f\left(E_{r}+\hbar \omega\right)\right]\left(E_{r}{ }^{2}+\Delta^{2}+\hbar \omega E_{r}\right)}{\hbar \omega\left(E_{r}{ }^{2}-\Delta^{2}\right)^{1 / 2}\left[\left(E_{r}+\hbar \omega\right)^{2}-\Delta^{2}\right]^{1 / 2}} d E_{r}+ \\
& \int_{-\Delta_{1}+\hbar \omega} \frac{\left[1-2 f\left(E_{r}-\hbar \omega\right)\right]\left(E_{r}{ }^{2}+\Delta_{1}{ }^{2}-\hbar \omega E_{r}\right)}{\left.\hbar \omega\left(E_{r}^{2}-\Delta_{1}\right)^{2}\right)^{1 / 2}\left[\left(E_{r}-\hbar \omega\right)^{2}-\Delta_{1}{ }^{2}\right]^{1 / 2}} d E_{r}- \\
& \int_{-\Delta_{2}}^{0} \frac{j\left[1-2 f\left(\Delta_{1}+j E_{i}\right)\left[\left(\Delta_{1}+j E_{i}\right)\left(\Delta_{1}^{2}+j E_{i}-\hbar \omega\right)+\Delta^{2}\right]\right.}{\hbar \omega\left[\left(\Delta_{1}+j E_{i}\right)-\Delta^{2}\right]^{1 / 2}\left[\left(\Delta_{1}+j E_{i}-\hbar \omega\right)^{2}-\Delta^{2}\right]^{1 / 2}} d E_{i}+ \\
& \int_{-\Delta_{2}}^{0} \frac{j\left[1-2 f\left(\Delta_{1}+j E_{i}\right)\left[\left(\Delta_{1}+j E_{i}\right)\left(\Delta_{1}^{2}+j E_{i}+\hbar \omega\right)+\Delta^{2}\right]\right.}{\hbar \omega\left[\left(\Delta_{1}+j E_{i}\right)-\Delta^{2}\right]^{1 / 2}\left[\left(\Delta_{1}+j E_{i}+\hbar \omega\right)^{2}-\Delta^{2}\right]^{1 / 2}} d E_{i}
\end{aligned}
$$

where $E_{r}$ and $E_{i}$ are, respectively, the real and imaginary parts of the complex quasiparticle excitation energy $E$ and $\sigma_{n}$ is the normal conductivity of the material at room temperature. The function,

$$
f(E)=\frac{1}{1+\exp (E / k T)},
$$

gives the Fermi-Dirac statistics, where $k$ is the Boltzmann's constant.

Niobium $\mathrm{Nb}$ has been widely used in the fabrication of the Superconductor-Insulator-Superconductor SIS mixer in millimeter/submillimeter radio receivers. Here, we employ $\mathrm{Nb}$ as the wall material of the circular waveguide. The critical temperature $T_{c}$, energy gap at $0 \mathrm{~K} 2 \Delta(0)$ and normal conductivity $\sigma_{n}$ of $\mathrm{Nb}$ are, respectively, given as $9.2 \mathrm{~K}, 3.05$ $\mathrm{meV}$ and $1.57 \times 10^{7} \mathrm{~S} / \mathrm{m}$ [13]. In an actual $\mathrm{Nb}$ film, the imaginary part of the energy gap $\Delta_{2}$ is found to be $10^{-4}$ of its real part $\Delta_{1}[9]$, [10].

\section{Propagation in a circular waveguide}

Fig. 1 depicts the structure of a circular waveguide. At the boundary of the waveguide where radius $r=a$, the tangential electric $E_{t}$ and magnetic $H_{t}$ fields in the waveguide are related to the electrical properties of the wall material by $[14]-[16]$

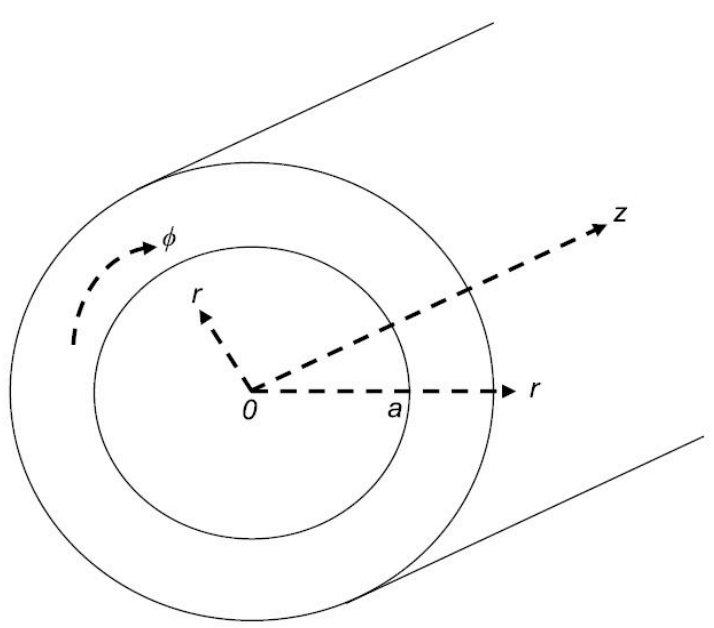

Figure 1: A circular waveguide.

$$
\frac{E_{t}}{H_{t}}=\sqrt{\frac{\mu \omega(\varepsilon \omega+j \sigma)}{(\varepsilon \omega)^{2}+\sigma^{2}}},
$$

where $\omega$ is the angular frequency, $\mu$ the permeability of the wall material and $\varepsilon$ is the permittivity of free space. For a superconducting waveguide, the conductivity $\sigma$ in (5) can be found by solving (3). By letting the determinant of the coefficients in (5) vanish; we obtain the following transcendental equation [5]

$$
\begin{aligned}
& {\left[j\left(k^{2}-k_{z}^{2}\right) \sqrt{\frac{\mu}{\varepsilon}}+\omega \mu \sqrt{k^{2}-k_{z}^{2}} \frac{J_{n}{ }^{\prime}(\chi)}{J_{n}(\chi)}\right] \times} \\
& {\left[j\left(k^{2}-k_{z}^{2}\right) \sqrt{\frac{\varepsilon}{\mu}}+\omega \varepsilon \sqrt{k^{2}-k_{z}^{2}} \frac{J_{n}{ }^{\prime}(\chi)}{J_{n}(\chi)}\right]=\left[\frac{n k_{z}}{a}\right]^{2}}
\end{aligned}
$$

where $J_{n}(\chi)$ denotes the Bessel function of the first kind, $J_{n}^{\prime}(\chi)$ its derivative, $\chi=\sqrt{a^{2}\left(k^{2}-k_{z}^{2}\right)}, n$ the order of the Bessel function, $k$ the wavenumber in free space and $k_{z}$ is the wave propagation constant. The propagation constant $k_{z}$ $=\beta_{z}-\mathrm{j} \alpha_{z}$ is a complex variable which comprises both the phase constant $\beta_{z}$ and attenuation constant $\alpha_{z}$. By extracting the imaginary part of $k_{z}$, the attenuation constant $\alpha_{z}$ can therefore be obtained.

\section{Results and discussion}

By numerically solving (6), the attenuation constant of a $\mathrm{Nb}$ circular waveguide with radius $a=8.1 \mathrm{~mm}$, operating at both room temperature and under the critical temperature $T_{c}$ at $T=4.2 \mathrm{~K}$ is calculated. Here, we have applied the Powell Hybrid root-searching algorithm to determine the roots of (6). To solve for the integrals in the complex gap energy $\Delta$ and the complex conductivity $\sigma$, we have applied the algorithms in the SLATEC mathematical library. Fig. 2 illustrates the overall attenuation of the dominant TE11 mode from frequency $f=0$ to $1.5 \mathrm{THz}$. It can be observed from the figure that the superconducting waveguide behaves differently at different range of frequencies. To analyze the behavior of the waveguide, we separate the attenuation into 
3 parts, i.e. the attenuation at (i) frequency $f$ below cutoff $f_{c}$ $\left(f<f_{c}\right)$, (ii) $f$ above cutoff but below the gap frequency $f_{g}\left(f_{c}\right.$ $\left.<f<f_{g}\right)$ and (iii) $f$ above $f_{g}\left(f>f_{g}\right)$. Fig. 3 depicts the attenuation of TE11 mode below the cutoff frequency $f_{c}$; whereas, Figs. 4 to 6 depicts the attenuation above $f_{c}$. As can be observed from the figures, at frequency $f$ below cutoff $f_{c}$, the attenuation in the superconducting waveguide is somewhat higher than that operating at normal state. On the other hand, when $f$ increases above $f_{c}$, the attenuation in the superconducting waveguide below the gap frequency $f_{g}$ decreases dramatically. As can be seen in Figs. 4 and 5, the attenuation in the superconducting waveguide turns out to be considerably lower than its other counterpart which is operating at room temperature. It is worthwhile noting that, although the attenuation we found here is low, it is finite. This is in contrast to the results shown in [4] and [5], where the attenuation above $f_{c}$ (but below $f_{g}$ ) is found to be infinitesimal. Since [4] and [5] (which applied MattisBardeen theory) assume that quasiparticles do not exist in a superconductor, while our method accounts for their presence, it is apparent that the attenuation found here is contributed by the quasiparticles at the intragap states. Ideally, a lossless waveguide behaves like a high pass filter where signals below the cutoff frequency $f_{c}$ cease to propagate through the waveguide. Above $f_{c}$ however, the attenuation in the lossless waveguide decreases sharply, allowing signals to propagate with negligible loss. Hence, it can be clearly seen from Figs. 2 to 5 that a superconducting circular waveguide behaves closer to a lossless waveguide than a normal waveguide. During superconducting state, the density of quasiparticles in the material is low. These quasiparticles are mainly those in the intragap states within the gap energy. Hence, energy loss due to collisions with the lattice structure is very low as well. This allows the superconducting waveguide to behave closer to a perfect waveguide, which is ideally lossless.

As the frequency $f$ increases above the gap frequency $f_{g}$, the photon energy exceeds the gap energy. With sufficient absorption of energy, Cooper pairs break into quasiparticles. Hence, the waveguide operating below the critical temperature $T_{c}$ starts to lose its superconductivity. As can be clearly seen from Fig. 6 , at $f$ above $f_{g}$, the attenuation in the waveguide at $4.2 \mathrm{~K}$ increases significantly. In fact, it surpasses that found in a normal waveguide. We attribute this phenomenon to the increase of random collision between the quasiparticles and the lattice structure at the wall, resulting in higher conduction loss in the waveguide. The results found in the superconducting circular waveguide agree with those of the superconducting rectangular waveguide. They, therefore, corroborate the findings in [12].

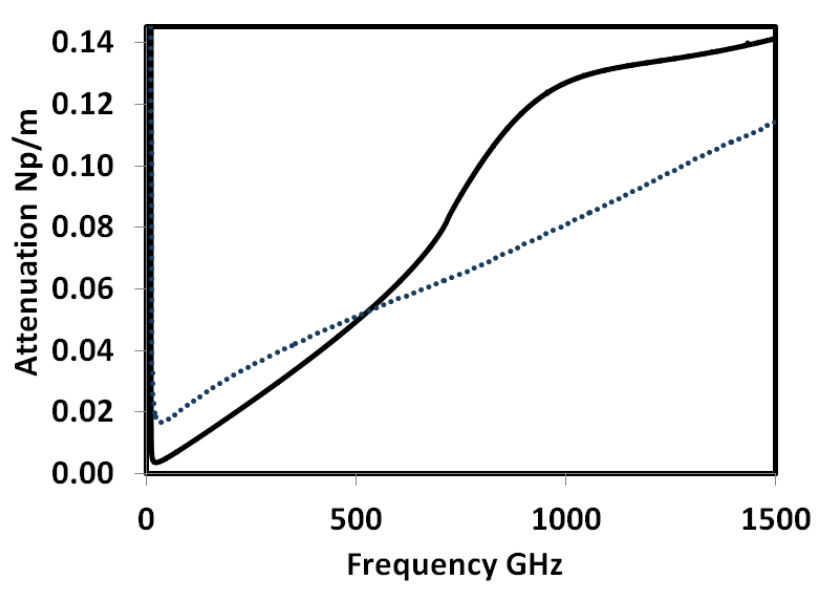

Figure 2: Attenuation of TE11 mode at frequency 0 to 1.5 $\mathrm{THz}$ in a $\mathrm{Nb}$ circular waveguide (with $8.1 \mathrm{~mm}$ radius), operating at $4.2 \mathrm{~K}$ (solid line) and room temperature (dotted line).

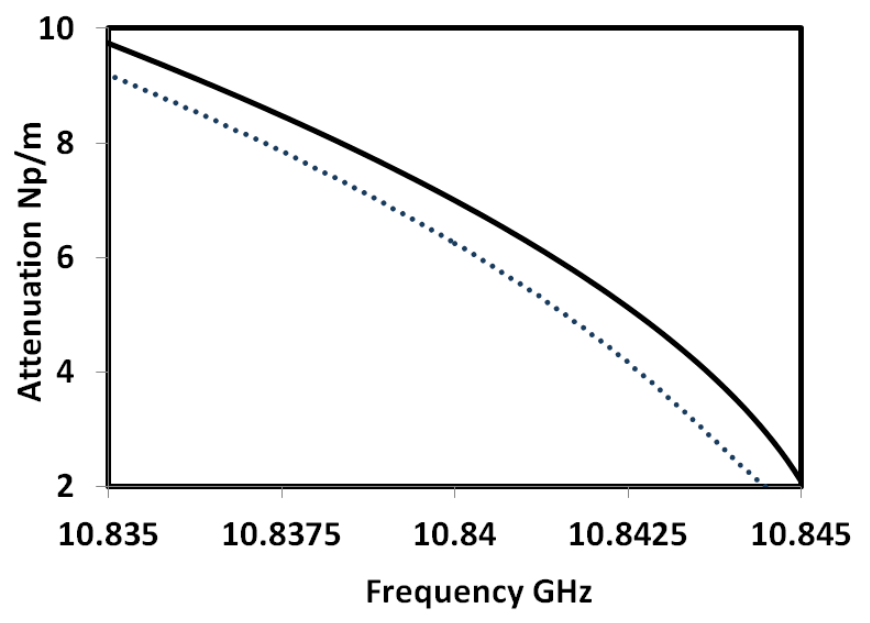

Figure 3: Attenuation of TE11 mode below cutoff in a $\mathrm{Nb}$ circular waveguide (with $8.1 \mathrm{~mm}$ radius), operating at $4.2 \mathrm{~K}$ (solid line) and room temperature (dotted line).

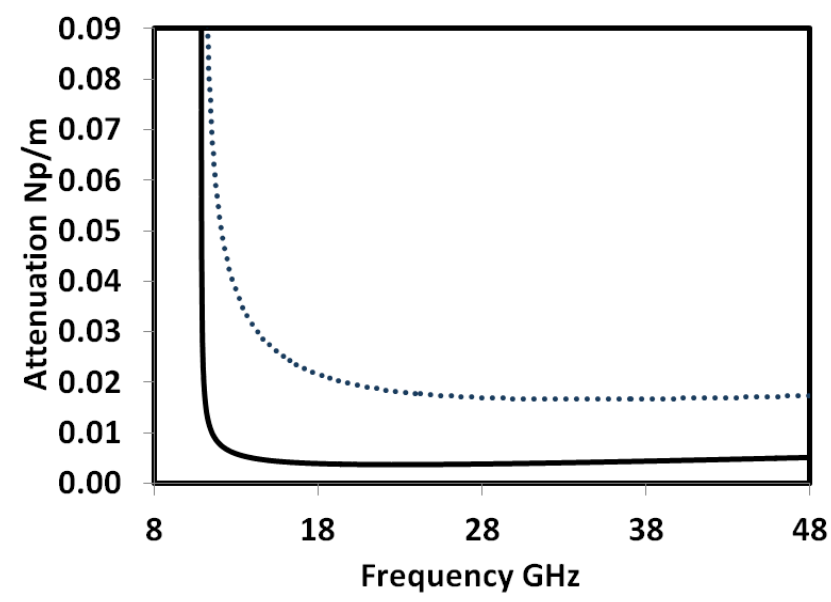

Figure 4: Attenuation of TE11 mode immediately above cutoff in a $\mathrm{Nb}$ circular waveguide (with $8.1 \mathrm{~mm}$ radius), operating at $4.2 \mathrm{~K}$ (solid line) and room temperature (dotted line). 


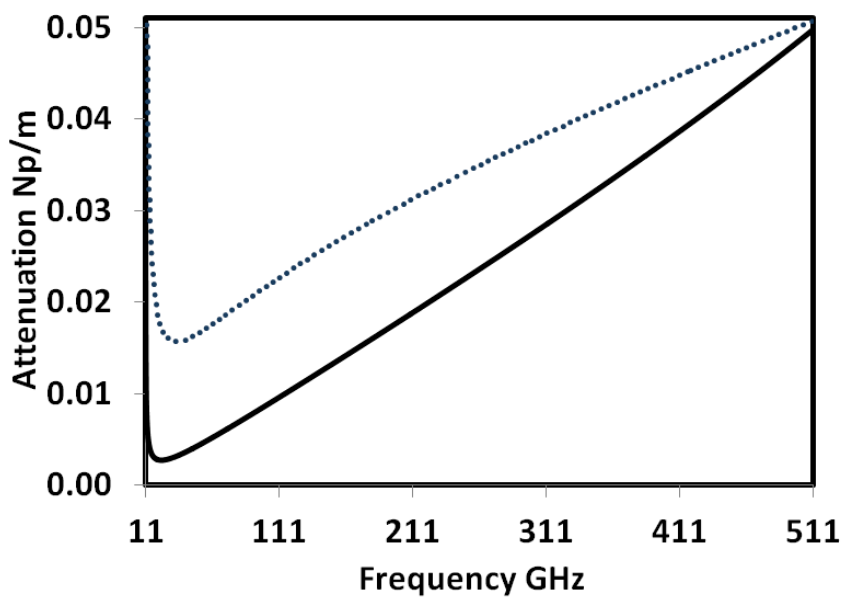

Figure 5: Attenuation of TE11 mode above cutoff but below the gap frequency in a $\mathrm{Nb}$ circular waveguide (with $8.1 \mathrm{~mm}$ radius), operating at $4.2 \mathrm{~K}$ (solid line) and room temperature (dotted line).

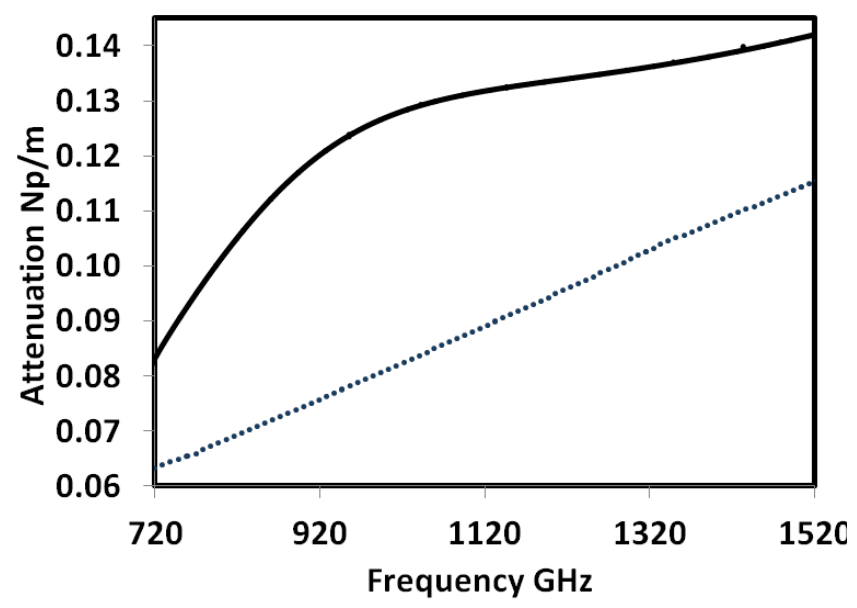

Figure 6: Attenuation of TE11 mode above the gap frequency in a $\mathrm{Nb}$ circular waveguide (with $8.1 \mathrm{~mm}$ radius), operating at $4.2 \mathrm{~K}$ (solid line) and room temperature (dotted line).

\section{Conclusion}

We have performed an analysis on superconducting circular waveguides based on the extended Mattis-Bardeen theory. In contrast with those found in literatures, our results show that the loss above cutoff in a superconducting waveguide is not infinitesimal. Although the loss turns out to be considerably lower than those in a normal waveguide, it is certainly finite. We attribute this phenomenon to the presence of quasiparticles in the intragap states within the gap energy. Above the gap energy, Cooper pairs break into quasiparticles. The waveguide operating below the critical temperature $T_{c}$ loses its superconducting characteristics. Hence, the loss increases significantly. Our results show that the loss of the waveguide eventually surpasses those found in a normal waveguide.

\section{Acknowledgements}

The authors thank Dr. Takashi Noguchi for his discussion. Part of this work has been supported by the FRGS grant (project: FRGS/2/2013/SG02/UTAR/02/1).

\section{References}

[1] K. C. Walker, J. W. Kooi, M. Chan, H. G. Leduc, P. L. Schaffer, J. E. Carlstrom, T. G. Phillips, A low-noise $492 \mathrm{GHz}$ SIS waveguide receiver, Int. J. of Infrared and Millimeter Waves 13: 785 - 798, 1992.

[2] K. H. Yeap, K. C. Yeong, C. Y. Tham, H. Nisar, Analysis of Energy Loss in Superconducting Waveguides, Biologically-Inspired Energy Harvesting through Wireless Sensor Technologies, IGI Global, Pennsylvania, 2016.

[3] J. H. Winters, C. Rose, High-Tc superconductors waveguides: Theory and applications, IEEE Transactions on Microwave Theory and Techniques 39: $617-623,1991$.

[4] G. Yassin, C. Y. Tham, S. Withington, Propagation in lossy and superconducting cylindrical waveguides, Proc. of the 14th Int. Symp. on THz Technology, Tucson, Arizona, pp. 516-519, 2003.

[5] K. H. Yeap, C. Y. Tham, K. C. Yeong, H. J. Woo, Wave propagation in lossy and superconducting circular wavguides, Radioengineering J. 19: 320 - 325, 2010.

[6] M. J. Wengler, Submillimeter-wave detection with superconducting tunnel diodes, Proc. of IEEE 80: 1810 $-1826,1992$.

[7] B. Mitrovic, L. A. Rozema, On the correct formula for the lifetime broadened superconducting density of states, J. Phys. Condens. Matter 20: 015215, 2008.

[8] T. Noguchi, T. Suzuki, A. Endo, T. Tamura, Contribution of the imaginary part of the superconducting gap energy on the SIS tunneling current, Physica C 469: 1585 - 1588, 2009.

[9] T. Noguchi, T. Suzuki, T. Tamura, Subgap tunneling current at low temperature in $\mathrm{Nb} / \mathrm{Al}-\mathrm{AlN} / \mathrm{Nb}$ SIS junctions, IEEE Transactions on Appl. Superconductivity 21: 756 - 759, 2011.

[10] T. Noguchi, M. Naruse, Y. Sekimoto, RF conductivity and surface impedance of a superconductor taking into account the complex superconducting gap energy, Phys. Proceedia 36: 318 - 323, 2012.

[11] T. Noguchi, M. Naruse, Y. Sekimoto, Contribution of quasiparticles in the subgap states to the surface impedance of superconductors, IEEE Transactions on Appl. Superconductivity 23: 1501404, 2013.

[12] K. H. Yeap, J. S. M. Teh, H. Nisar, K. C. Yeong, K. Hirasawa, Attenuation in superconducting rectangular waveguides, Frequenz J. of RF-Engineering and Telecommunications 69: 111 - 117, 2015.

[13] P. L. Kautz, Picosecond pulses on superconducting striplines, J. of Appl. Phys. 49: 308 - 314, 1978. 
[14] K. H. Yeap, C. Y. Tham, K. C. Yeong, K. H. Yeap, A simple method for calculating attenuation in waveguides, Frequenz J. of RF-Engineering and Telecommunications 63: 236-240, 2009.

[15] K. H. Yeap, C. Y. Tham, K. C. Yeong, E. H. Lim, Full wave analysis of normal and superconducting microstrip transmission lines, Frequenz J. of $R F$ Engineering and Telecommunications 64: 56 - 66, 2010.

[16] K. H. Yeap, C. Y. Tham, G. Yassin, K. C. Yeong, Attenuation in rectangular waveguides with finite conductivity walls, Radioengineering $J$. 20: $472-478$, 2011. 